ThVOVed

Laura SORMANI LE BOURHIS ${ }^{1}$, Hélène TAQUIN ${ }^{2}$, Rana MHAIDLY ${ }^{1}$, Els VERHOYEN ${ }^{1}$, Marjorie HEIM ${ }^{1}$, Claire REGAZZETTI ${ }^{1}$, Meri K. TULIC $^{1}$, Yann CHELI ${ }^{1}$, Nicolas NOTTET ${ }^{1}$,

Gian Marco DE DONATIS ${ }^{1}$, Anne-Sophie DABERT GAY ${ }^{4}$, Delphine DEBAYLE ${ }^{4}$, Nathalie CARDOT LECCIA ${ }^{3}$, Henri MONTAUDIE ${ }^{1}$, Stéphane ROCCHI ${ }^{1}$ \& Thierry PASSERON ${ }^{1,2}$

1 Université Côte d'Azur. Centre Méditerranéen de Médecine Moléculaire (C3M) - UNS - INSERM U1065 - Nice - France

\title{
Introduction
}

Melanogenesis is a complex and tightly regulated process. In a recent transcriptome analysis performed in lesional skin compared to non lesional skin of vitiligo patients, we have identified a new gene called CLEC12B. The decreased expression of CLEC12B in skin lacking melanocytes compared to pigmented skin is comparable to the one observed for key melanogenic enzymes TYR, DCT or TYRP1 suggesting that CLEC12B could be an important

melanocytic gene. CLEC12B is a member of the C-type lectin family which are transmembrane receptors that possess an ITIM domain recruiting phosphatases. So far, few data are available on this gene that has only been shown in myeloid cells. Ligand and downstream signaling of CLEC12B are unknown and to date, no link has been reported between CLEC12B and pigmentation.

\section{Background results}

1. Transcriptome analysis of vitiligo skin reveals CLEC12B as a new melanocyte specific biomarker

Clinical study design

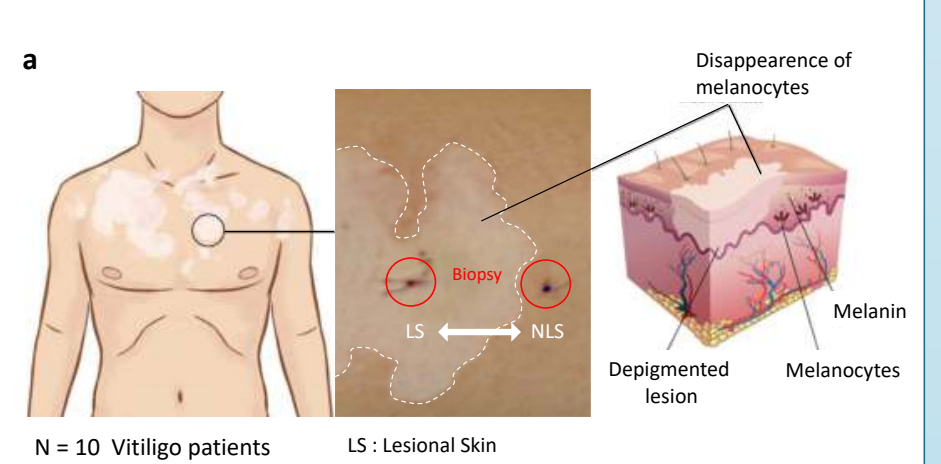
$N=10$ Vitiligo patients $\begin{gathered}\text { Is: Lesional Isin } \\ \text { Nis: : Non Lesional Skin }\end{gathered}$

b

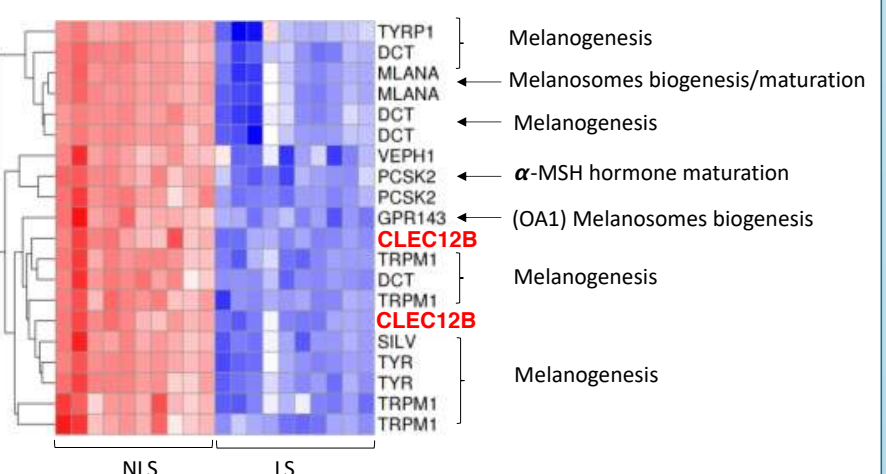

Figure 1 Transcriptome analysis of vitiligo skin highlight the disappearance of melanocytes and reveals CLEC12B (a) 4-mm-skin biopsy was taken from patient with vitiligo in located in the same area but at least $3 \mathrm{~cm}$ from the

depigmented lesion.
(b) Transcriptomic analysis highlights top 20 downregulated genes in lesional skin compared to non-lesional skin.

\section{Objectives}

To examine whether human melanocytes express CLEC12B and

if CLEC12B has effect on melanogenesis.

\section{CLEC12B :}

\section{C-type Lectin Like Receptor}

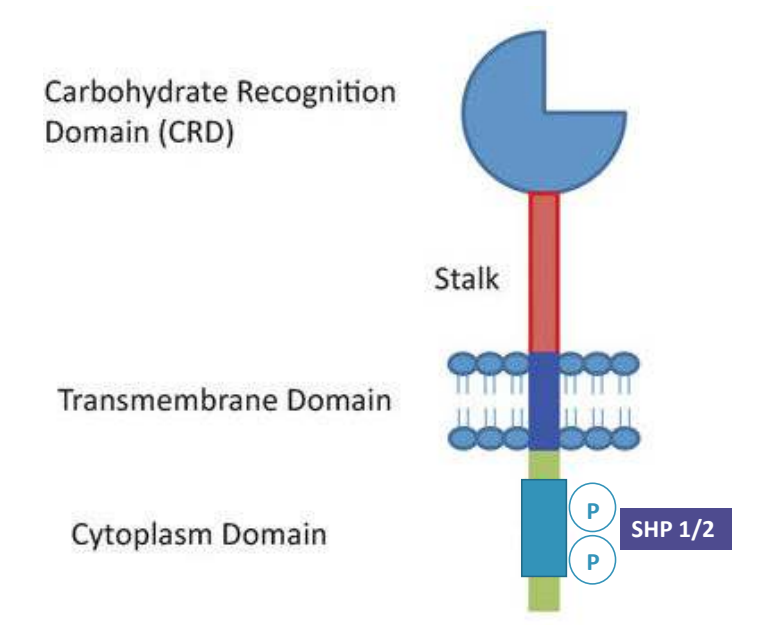

Member of the C-type lectin family of transmembrane receptors

CLEC12B possesses an ITIM domain which can recruit phosphatases like SHP1 or SHP-2 upon phosphorylation.

- Ligand and downstream signalling of CLEC12B are largely unknown.

\section{Results}

2. CLEC12B is expressed by skin melanocytes

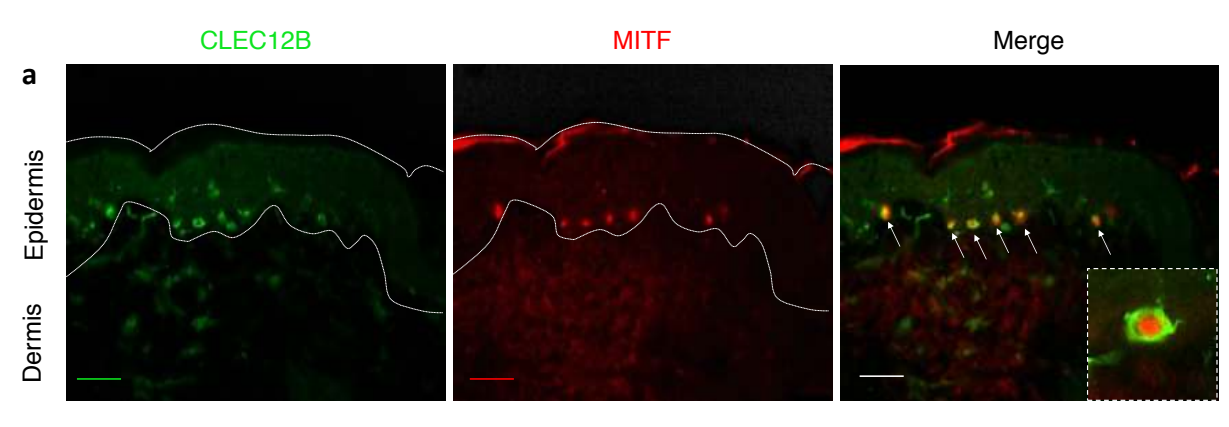

Figure 2 (a) Immunofluorescence staining performed on human skin sample taken from abdominoplasty shows a colocalization (yellow) of CLEC12B (green) with MITF. (b) CLEC12B mRNA relative quantity was examined by GPCR in 4. CLEC12B modulates melanogenesis in vitro

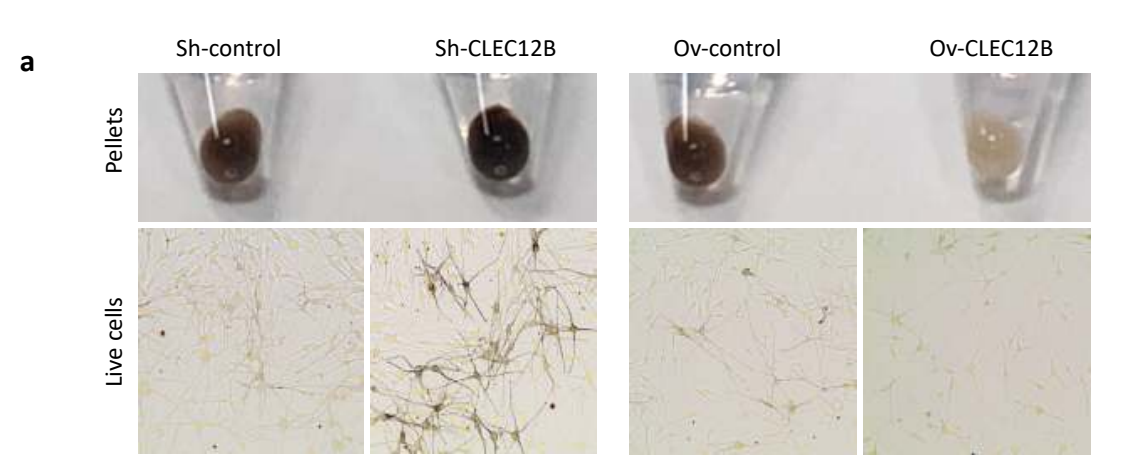

Figure 4 Lentiviral infection was used to silence (Sh-CLEC12B) or overexp
transmitted light microscopy was semi-quantitated using a melanin assay

5. CLEC12B modulates pigmentation in reconstructed human skin

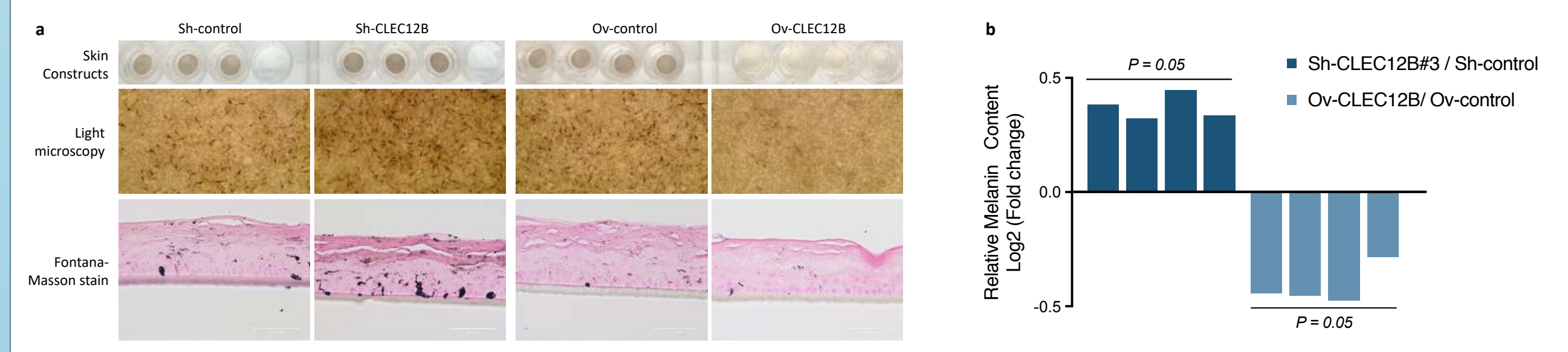

Figure 5 Reconstructed skins were composed of keratinocytes cultured in presence of phototype III/IV melanocytes transduced with lentivirus to silence (Sh-CLEC12B) or to overexpress CLEC12B (OVCLEC12B). (a) The variation of pigmentation was observed in skin constructs and by transmitted light microscopy. Melanin pigments were visualized with Fontana-Masson and Eosin stain. (b) Melanin production was assessed using a melanin assay.

6. CLEC12B regulates melanogenesis pathway by recruiting SHP-1 and SHP-2

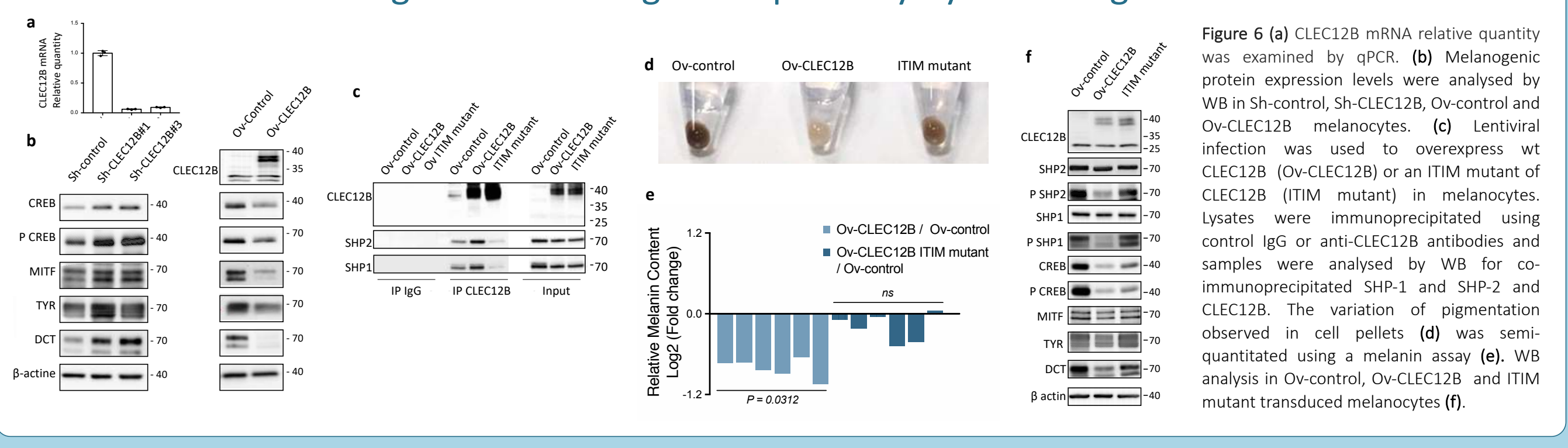

\section{Conclusion}

CLEC12B appears as a new melanocytic gene that strongly modulates melanogenesis by recruiting

SHP-1 and SHP-2. These results not only provides novel insights in the understanding of the melanocyte biology and regulation of melanogenesis but also supports CLEC12B as a promising target for the development of melanogenic agents in the clinic and cosmetic fields. 